\title{
Non-contact Crack Detection in Metals Using a Cutoff-Cavity Probe
}

\author{
Ki-Chai Kim ${ }^{1, *} \cdot$ Jong-Woo Kim² $\cdot$ Jae-Yong Kwon ${ }^{3} \cdot$ No-Weon Kang ${ }^{3}$
}

\begin{abstract}
This paper presents a non-contact method for the detection of surface cracks in metal materials through a forced-resonance microwave method (FRMM) using a cutoff cavity-backed narrow slot as a crack detection probe without using a vector network analyzer (VNA) at microwave frequencies. The FRMM uses the deviations in the ammeter or voltmeter readings of the forcefully obtained resonance of a cutoff-cavity probe for a metal material with or without cracks. The cutoff cavity-backed narrow slot on metal with no cracks produces a series resonance (maximum current) or a parallel resonance through an external control element located on a post inside the cutoff cavity. Cracks were detected by a change in this forced-resonance state (maximum current) when the cutoff-cavity probe was scanned over a crack. The characteristic crack signal was derived from the resonance current deviation on the ammeter located on a post inside the cavity probe. Galerkin's method of moments was used to obtain a forced-resonance state from which the crack signal of the FRMM was calculated. The experimental measurements for non-contact (remote or lift-off) crack detection are also presented.
\end{abstract}

Key Words: Cutoff-Cavity, Forced-Resonance, Microwave Method, Non-contact Crack Detection, Non-destructive Testing.

\section{INTRODUCTION}

Metal failure or fatigue usually begins at the surface. Several prominent non-destructive testing (NDT) techniques can be used to detect surface cracks in metal. These techniques include dye penetrant testing, ultrasonic testing, eddy current testing, acoustic emission testing, and radiographic testing [1-3]. In addition, several studies have shown that the detection and sizing of surface cracks in metals can be achieved non-destructively using microwave and $\mathrm{mm}$-wave frequencies [4-9]. Microwave techniques have certain advantages when detecting hairline stress or fatigue cracks. An open-ended waveguide operating at $20 \mathrm{GHz}$ was used as a sensor to detect smaller surface cracks, such as fatigue cracks, through changes in the reflection coefficient [4]. This method, however, can be affected easily by noise, air gaps, and other factors [5]. Several critical parameters of the open-ended waveguide technique were investigated to resolve this problem $[6,7]$. The open-ended waveguide technique needs to be used at higher frequencies to achieve high spatial resolution [8]. Open-ended coaxial probes and near-field sensors using resonant probes have been used for crack detection in metallic materials $[9,10]$. The resonant probes provide many advantages over other probes based on measurements of the changes in the reflection coefficient. Recently, several techniques with resonant probes have been reported on, including those using a microstrip linear resonator, quarterwavelength microstrip line, dual-behavior resonator filter, and

\footnotetext{
Manuscript received October 23, 2020 ; Revised December 19, 2020 ; Accepted January 7, 2021. (ID No. 20201023-169J)

${ }^{1}$ Department of Electrical Engineering, Yeungnam University, Gyeongsan, Korea.

${ }^{2}$ EMC Team, Korea Marine Equipment Research Institute (KOMERI), Busan, Korea.

${ }^{3}$ Center for Electromagnetic Metrology, Korea Research Institute of Standards and Science (KRISS), Daejeon, Korea.

"Corresponding Author: Ki-Chai Kim (e-mail: kckim@ynu.ac.kr)

Current affiliation for author, Jae-Yong Kwon, is: Science of Measurement, University of Science and Technology, Daejeon, Korea.
}

This is an Open-Access article distributed under the terms of the Creative Commons Attribution Non-Commercial License (http://creativecommons.org/licenses/by-nc/4.0) which permits unrestricted non-commercial use, distribution, and reproduction in any medium, provided the original work is properly cited.

(c) Copyright The Korean Institute of Electromagnetic Engineering and Science. 
complementary split-ring resonator [11-14]. A more general review of state-of-the-art NDT techniques using microwave technologies has been provided elsewhere [15]. In these techniques, the metal surface is scanned by a waveguide probe, while its reflection characteristics are monitored using a slotted guide and a diode detector, or with a network analyzer. The crack detection and sizing in these techniques are done by analyzing the overall reflection coefficient of the incident field at different crack positions beneath the waveguide probe aperture. On the other hand, cutoff-cavity probes have been proposed for crack detection in metallic materials [16]. The crack detection in this technique is done by analyzing the resonant current deviation of the cutoffcavity probe at different crack positions beneath the cutoff-cavity probe aperture. This method is called the forced-resonance microwave method (FRMM). The FRMM uses the current deviation from the resonance state for the cutoff-cavity probe without a crack and with a crack. Therefore, knowledge of the current characteristics of the cutoff-cavity probe is needed to obtain crack signals [17].

This paper presents a method of remote crack detection using a cutoff-cavity probe with a FRMM. This study used a cutoff cavity-backed narrow slot probe based on previous work with the FRMM for crack detection in metal materials [16]. In [16], cavity-backed wide slot apertures were used, and the aperture field distribution was expanded in cavity mode. In this paper, however, cavity-backed narrow slots were used, and the slot could be treated as a thin-wire approximation. The advantage of using the cavity-backed narrow slot aperture instead of the wide slot aperture is the increased crack location detection accuracy. In addition, the thin-wire approximation also has the advantage of being able to easily compute the magnetic current distributions on the crack and narrow slot in the cutoff-cavity probe. This approximation is valid for microwave frequencies on a thin crack and cavity-backed narrow slot. Galerkin's method of moments was used to obtain the forced-resonance state from which the crack signal was calculated. The cutoff-cavity probe produces forced resonance by adjusting a control element. Forced resonance can be divided into two types: series resonance (SR) and parallel resonance $(\mathrm{PR})[16,17]$. SR gives rise to a maximum current, while PR shows sluggish gradient currents. The minimum current occurs at the non-resonance (NR) state via the control element. The FRMM mainly uses SR, but PR and NR can also be used for crack detection. However, PR and NR are not as advantageous as SR [17]. This study examined noncontact crack detection signals using forced-resonance at SR. This method can be used to detect hairline and wide cracks with a single microwave frequency. The cutoff-cavity probe was designed and tested numerically and experimentally, and the crack characteristic signals were detected using the fabricated prototype cutoff-cavity probe.

\section{SYSTEM DESCRIPTION}

Fig. 1 shows the geometry of the system of remote crack detection by cutoff-cavity probes using the FRMM. Fig. 1(a) and (b) correspond to reference setting on a non-cracked metal (corresponding to the shorting plate) and the remote (lift-off or noncontact) crack detection, respectively. The detection system consists of four parts: (1) the cutoff cavity-backed narrow slot probe (called a cutoff-cavity probe) and two internal posts, (2) the external control element for cavity resonance control, (3) the generator, and (4) the detector for reading the forced-resonance characteristics and crack characteristic signals. An ammeter or voltmeter can be used as the detector. In this study, an ammeter was chosen.

The cutoff-cavity probe has a narrow slot with a width of $W_{\mathrm{s}}$ and length of $L_{\mathrm{s}}$ and is in a small conducting flange with $z=0$. This is backed by a conducting rectangular cutoff cavity of depth $c$. A crack with width $W$, length $L$, and depth $d$ is located in the metal. The cutoff-cavity dimensions were chosen such that a cross-section of cavity $a \times b$ corresponds to the cutoff condition for a waveguide of the same cross-section when the cavity is empty. For this reason, it is called the cutoff cavity. In the cutoff cavity, a feed post (\#1) with a radius of $r_{1}$ is located at $x=a / 2$ and $z=s_{1}$.

The external control element $j X_{1}$ (acting as a reactance element)

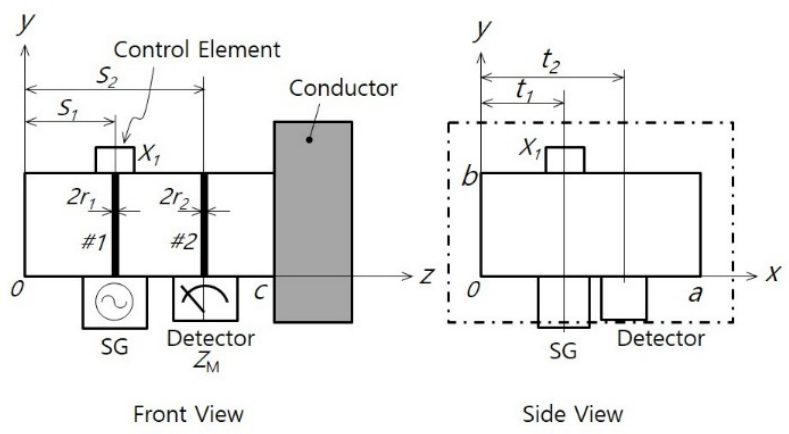

(a)

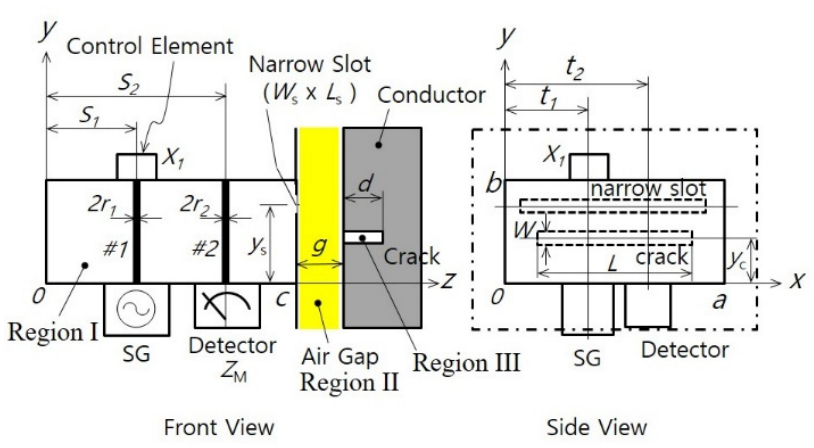

(b)

Fig. 1. Cutoff cavity-backed narrow slot for remote crack detection: (a) reference setting on a non-cracked metal (corresponding to shorting plate) and (b) remote (lift-off or non-contact) crack detection. 
is connected to the top of the feed post to obtain the desired forced resonance in the cutoff-cavity probe. For example, commercial products such as coaxial sliding shorts (Model 1909C2 at 0.8-4 GHz; Maury Microwave Corp., Ontario, CA, USA) may be used as the control element. Alternatively, it can be constructed using semi-rigid coaxial cables with SMA connectors. Adjusting the length of the control element changes the reactance value, which changes the characteristics of the cutoff-cavity probe.

An ammeter is connected to the bottom of a parasitic post (\#2) with a radius of $r_{2}$ at $y=0, x=a / 2$, and $z=s_{2}$. The ammeter obtains the desired forced resonance and measures the output crack signals. A voltage of $V_{1}$ is applied at $y=0, x=a / 2$, and $z=s_{1}$, and the control element $j X_{1}$ is connected at $y=b, x=a / 2$, and $z=s_{1}$. Each parameter referenced in Fig. 1 is listed in Table 1.

Two steps (preparation and detection stages) are necessary to detect surface crack signals in the air gap layer-covered metals using the FRMM.

\section{THEORETICAL ANALYSIS OF THE FRMM}

\section{Step 1: Preparation Stage (Reference Setting)}

The procedure for using the FRMM and the cutoff-cavity probe to detect surface cracks has been detailed elsewhere [16]. While reference setting, the cutoff-cavity probe and metal are in perfect contact. According to this procedure, the cutoff-cavity probe with a metal shorting plate (in the absence of a crack) uses the control element, $j X_{1}$, which is connected to the top of feed post \#1, to resonate forcefully in the cutoff cavity.

Table 1. Geometric parameters

\begin{tabular}{lcc}
\hline \multicolumn{1}{c}{ Parameter } & Symbol & Value (mm) \\
\hline Cavity width & $a$ & 40 \\
Cavity height & $b$ & 20 \\
Cavity depth & $c$ & 80 \\
Slot width & $W_{s}$ & 5 \\
Slot length & $L_{s}$ & 40 \\
Slot center position & $y_{s}$ & 10 \\
$x$ position of the feed post\#1 & $t_{1}$ & 20 \\
$x$ position of the parasitic post\#2 & $t_{2}$ & 20 \\
$z$ position of the feed post\#1 & $S_{1}$ & 30 \\
$z$ position of the parasitic post\#2 & $S_{2}$ & 60 \\
Radius of the feed post\#1 & $r_{1}$ & 1 \\
Radius of the parasitic post \#2 & $r_{2}$ & 1 \\
Crack center position & $y_{c}$ & Variable \\
Crack width & $W$ & 1 \\
Crack length & $L$ & 40 \\
Crack depth & $d$ & 1 \\
External reactance & $X_{1}$ & Variable \\
Detector impedance & $Z_{M}$ & $5 \mathrm{k} \Omega$ \\
\hline
\end{tabular}

For the cutoff-cavity probe with a shorting plate, as shown in Fig. 1(a), integral equations for current distributions can be derived if the cutoff-cavity probe is fed by a delta gap generator as the voltage source in Step 1. The simultaneous integral equations for the unknown electric currents, $\bar{J}_{1}$ and $\bar{J}_{2}$, on feed post \#1 and parasitic post \#2 in Step 1 are as follows:

$$
\begin{aligned}
& \oiint_{S_{1}^{\prime}} \overline{\bar{K}}_{e e 11}^{\mathrm{I}} \cdot \bar{J}_{1} d S_{1}^{\prime}+\oiint_{S_{2}^{\prime}} \overline{\bar{K}}_{e e 12}^{\mathrm{I}} \cdot \bar{J}_{2} d S_{2}^{\prime} \\
& =\hat{y} V_{1} \delta(y)+\hat{y} j X_{1} I_{1}(b) \delta(y-b) \\
& \oiint_{S_{1}^{\prime}} \overline{\bar{K}}_{e e 21}^{\mathrm{I}} \cdot \bar{J}_{1} d S_{1}^{\prime}+\oiint_{S_{2}^{\prime}} \overline{\bar{K}}_{e e 22}^{\mathrm{I}} \cdot \bar{J}_{2} d S_{2}^{\prime} \\
& =\hat{y} Z_{M} I_{2}(0) \delta(y)
\end{aligned}
$$

where kernels $\overline{\bar{K}}_{e e i j}^{\mathrm{I}}$ are the dyadic Green's functions that denote the electric fields yielded from a unit of electric current; $\delta(*)$ is the delta function, and $I_{1}(b)$ and $I_{2}(0)$ are the currents at port 2 and the ammeter, respectively. $Z_{M}$ is the impedance of the ammeter. For convenience and simplicity, the current $I_{2}(0)$ at the ammeter-loading position will be expressed as $I_{2 \mathrm{~L}}$. The details of the cavity resonance characteristics are discussed in [17].

\section{Step 2: Crack Detection Stage}

According to the aforementioned procedure, the next step is the detection of crack signals. Fig. 2 depicts the cutoff-cavity probe being scanned toward a crack to detect the crack in the metal material. Fig. 2(a) and (b) demonstrate reference setting (in the preparation stage and in the absence of a crack) and remote crack

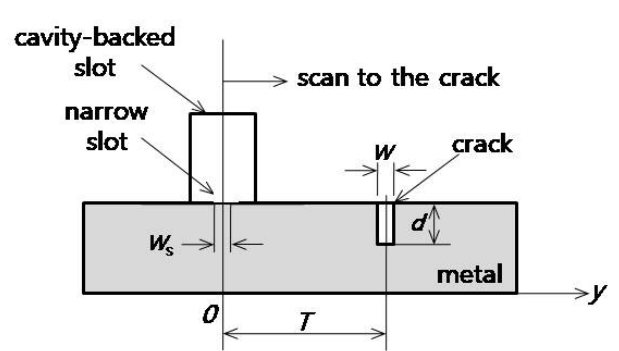

(a)

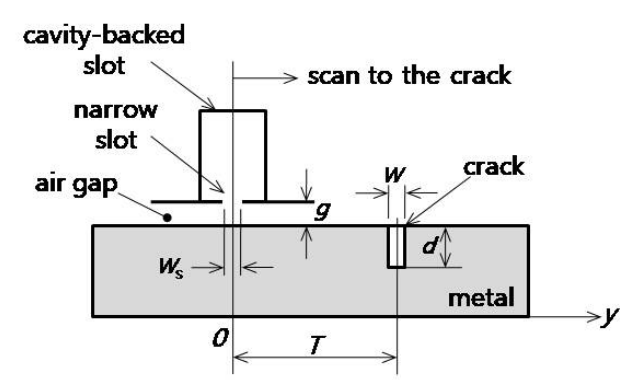

(b)

Fig. 2. Geometry of scanning for crack characteristic signal detection: (a) reference setting on a non-cracked metal and (b) remote (lift-off or non-contact) crack detection. 
detection (lift-off or non-contact detection), respectively. The cutoff-cavity probe detects a characteristic crack signal when there is a crack inside the narrow slot of the cutoff cavity. $T$ is the scanning position measured from the crack center to the center of the cavity's narrow slot.

Fig. 1(b) shows the geometry of a crack and a cutoff-cavity probe when the crack length $L$ is parallel to the broad dimensions of the cavity slot. The cutoff-cavity probe can scan the surface to detect cracks in the metal, and the ammeter records the detector output current on parasitic post \#2. If a crack is outside the narrow slot of the cutoff cavity, the detector output current reading will be the same as the detector output current in the short-circuit case of Step 1 (when there is no crack). In this case, the resonant current would be the same as the value detected in the case of a short circuit. On the other hand, if the cutoff cavity had a crack inside the narrow slot, then the detector output current would be different from the short-circuit case in Step 1, and the characteristic crack signal would be detected as a result.

The integral equations are derived by dividing the cutoff-cavity probe with the air gap layer (corresponding to the dielectric layer) covered crack into three regions: a cavity probe (region I), an air gap layer (region II), and a crack (region III), as shown in Fig. 1(b). The simultaneous integral equations for the unknown electric currents on the feed and parasitic post inside the cavity and the unknown magnetic currents $\bar{M}_{1}\left(=\hat{\mathrm{z}} \times \bar{E}_{\mathrm{a} 1}\right)$ on the cavity slot aperture and $\bar{M}_{2}\left(=\hat{\mathrm{z}} \times \bar{E}_{\mathrm{a} 2}\right)$ on the crack aperture, where $\bar{E}_{\mathrm{a} 1}$ and $\bar{E}_{\mathrm{a} 2}$ are the aperture electric field in the cavity slot aperture and crack aperture, respectively, can be written as follows:

$$
\begin{gathered}
\oiint_{S_{1}^{\prime}} \overline{\bar{K}}_{e e 11}^{\mathrm{I}} \cdot \bar{J}_{1} d S_{1}^{\prime}+\oiint_{S_{2}^{\prime}} \overline{\bar{K}}_{e e 12}^{\mathrm{I}} \cdot \bar{J}_{2} d S_{2}^{\prime} \\
+\iint_{S_{\mathrm{a} 1}^{\prime}} \overline{\bar{K}}_{e m 1 p}^{\mathrm{I}} \cdot \bar{M}_{1} d S_{\mathrm{a} 1}^{\prime} \\
=\hat{y} V_{1} \delta(y)+\hat{y} j X_{1} I_{1}(b) \delta(y-b) \\
\oiint_{S_{1}^{\prime}} \overline{\bar{K}}_{e e 21}^{\mathrm{I}} \cdot \bar{J}_{1} d S_{1}^{\prime}+\oiint_{S_{2}^{\prime}} \overline{\bar{K}}_{e e 22}^{\mathrm{I}} \cdot \bar{J}_{2} d S_{2}^{\prime} \\
+\iint_{S_{\mathrm{a} 1}^{\prime}} \overline{\bar{K}}_{e m 2 p}^{\mathrm{I}} \cdot \bar{M}_{1} d S_{\mathrm{a} 1}^{\prime} \\
=\hat{y} Z_{M} I_{2}(0) \delta(y) \\
\hat{\mathrm{z} \times}\left\{\oiint_{S_{1}^{\prime}} \overline{\bar{K}}_{h e 1 p}^{\mathrm{I}} \cdot \bar{J}_{1} d S_{1}^{\prime}+\oiint_{S_{2}^{\prime}} \overline{\bar{K}}_{h e 2 p}^{\mathrm{I}} \cdot \bar{J}_{2} d S_{2}^{\prime}\right. \\
+\iint_{S_{\mathrm{a} 1}^{\prime}}\left(\overline{\bar{K}}_{h m p p}^{\mathrm{I}}+\overline{\bar{K}}_{h m p p}^{\mathrm{II}}\right) \cdot \bar{M}_{1} d S_{\mathrm{a} 1}^{\prime} \\
\left.\quad-\iint_{S_{\mathrm{a} 2}^{\prime}} \overline{\bar{K}}_{h m p c}^{\mathrm{II}} \cdot \bar{M}_{2} d S_{\mathrm{a} 2}^{\prime}\right\}=0
\end{gathered}
$$

$$
\begin{aligned}
& \hat{\mathrm{z}} \times\left\{\iint_{S_{\mathrm{a} 1}^{\prime}} \overline{\bar{K}}_{h m c p}^{\mathrm{II}} \cdot\left(-\bar{M}_{1}\right) d S_{\mathrm{a} 1}^{\prime}\right. \\
& \left.+\iint_{S_{\mathrm{a} 2}^{\prime}}\left(\overline{\bar{K}}_{h m c c}^{\mathrm{II}}+\overline{\bar{K}}_{h m c c}^{\mathrm{III}}\right) \cdot \bar{M}_{2} d S_{\mathrm{a} 2}^{\prime}\right\}=0
\end{aligned}
$$

where superscripts I and II denote regions I and II, and subscripts $1,2, a 1$ (corresponding to probe aperture, $p$ ), and $a 2$ (corresponding to crack aperture, $c$ ) represent the feed post \#1, parasitic post \#2, the cavity probe slot aperture, and the crack aperture, respectively. In addition, $\hat{z}$ is the unit vector of the $z$-direction. $d S_{\mathrm{a} 1}^{\prime}$ and $d S_{\mathrm{a} 2}^{\prime}$ denote the element of the area on the surface of the probe slot aperture and the crack aperture, respectively. In addition, the kernels are as follows:

$$
\begin{gathered}
\overline{\bar{K}}_{e e i j}^{\mathrm{I}}=\frac{1}{j \omega \varepsilon_{0}}\left(\overline{\bar{I}} k_{0}^{2}+\nabla \nabla\right) \cdot \overline{\bar{G}}_{e i j}^{\mathrm{I}}, i, j=1 \text { or } 2 \\
\overline{\bar{K}}_{\text {emip }}^{\mathrm{I}}=-\nabla \times \overline{\bar{G}}_{\text {mip }}^{\mathrm{I}}, \quad i=1 \text { or } 2 \\
\overline{\bar{K}}_{\text {heip }}^{\mathrm{I}}=\nabla \times \overline{\bar{G}}_{\text {eip }}^{\mathrm{I}}, \quad i=1 \text { or } 2 \\
\overline{\bar{K}}_{h m p p}^{\mathrm{I}, \mathrm{II}}=\frac{1}{j \omega \mu_{0}}\left(\overline{\bar{I}} k_{0}^{2}+\nabla \nabla\right) \cdot \overline{\bar{G}}_{m p p}^{\mathrm{I}, \mathrm{II}} \\
\overline{\bar{K}}_{h m p c}^{\mathrm{II}}=\frac{1}{j \omega \mu_{0}}\left(\overline{\bar{I}} k_{0}^{2}+\nabla \nabla\right) \cdot \overline{\bar{G}}_{m p c}^{\mathrm{II}} \\
\overline{\bar{K}}_{h m c p}^{\mathrm{II}}=\frac{1}{j \omega \mu_{0}}\left(\overline{\bar{I}} k_{0}^{2}+\nabla \nabla\right) \cdot \overline{\bar{G}}_{m c p}^{\mathrm{II}} \\
\overline{\bar{K}}_{h m c c}^{\mathrm{II}, \mathrm{III}}=\frac{1}{j \omega \mu_{0}}\left(\overline{\bar{I}} k_{0}^{2}+\nabla \nabla\right) \cdot \overline{\bar{G}}_{m c c}^{\mathrm{II}, \mathrm{III}}
\end{gathered}
$$

where $\overline{\bar{I}}$ is the unit dyadic, $\overline{\bar{G}}_{e i j}^{\mathrm{I}}$ and $\overline{\bar{G}}_{e i p}^{\mathrm{I}}$ are the dyadic Green's functions that denote the electric type from the unit electric current in region $\mathrm{I}$, and $\overline{\bar{G}}_{m i j}^{\mathrm{I}, \mathrm{II}}(i=1$ or $2, j=p$ or $c)$ are the dyadic Green's functions that denote the magnetic type from the unit magnetic current in region I and II. $\overline{\bar{G}}_{m c c}^{\mathrm{III}}$ is the dyadic Green's function that denotes the magnetic type from the unit magnetic current in region III.

The air gap layer (region II) is represented by a parallel-plate waveguide. The Green's function for region II can be expressed as follows by using the principle of images:

$$
\overline{\bar{G}}_{m i j}^{\mathrm{II}}=\overline{\bar{I}} \sum_{n=-\infty}^{n=\infty}\left(\frac{e^{-j k_{2} R_{n i j}^{+}}}{4 \pi R_{n i j}^{+}}+\frac{e^{-j k_{2} R_{n i j}^{-}}}{4 \pi R_{n i j}^{-}}\right),
$$

where the distance $R_{n i j}^{ \pm}$between the source and the field points is given by

$$
R_{n i j}^{ \pm}=\sqrt{\left(x_{i}-x_{j}^{\prime}\right)^{2}+\left(\frac{W_{j}}{4}\right)^{2}+\left(z_{i} \mp z_{j}^{\prime}-2 n g\right)^{2}},
$$

where $x_{i}$ and $z_{i}$ are the coordinates of the field points, $x_{j}^{\prime}$ and $z_{j}^{\prime}$ represent the source points, and $W_{j}=W_{s}$ for $j=p$ and $W$ for $j=c$.

To solve the simultaneous integral equations with the method of moments for the unknowns, $\bar{J}_{1}, \bar{J}_{2}, \bar{E}_{a 1}$, and $\bar{E}_{a 2}$ were expanded 
as follows:

$$
\begin{gathered}
\bar{J}_{1}(y)=\hat{y} \sum_{k=0}^{K} a_{1 k} \cos \left(\frac{k \pi y}{b}\right) \\
\bar{J}_{2}(y)=\hat{y} \sum_{l=0}^{L} a_{2 l} \cos \left(\frac{l \pi y}{b}\right) \\
\bar{E}_{a 1}\left(x^{\prime}, y^{\prime}\right)=\hat{y} \sum_{p=1}^{P} e_{1 p} F_{p}\left(x^{\prime}\right) H_{1}\left(y^{\prime}\right) \\
\bar{E}_{a 2}\left(x^{\prime}, y^{\prime}\right)=\hat{y} \sum_{q=1}^{Q} e_{2 q} F_{q}\left(x^{\prime}\right) H_{2}\left(y^{\prime}\right) \\
F_{p}\left(x^{\prime}\right)=\left\{\begin{array}{l}
\frac{\sin k_{0}\left(x^{\prime}-x^{\prime}{ }_{p-1}\right)}{\sin k_{0} \Delta x}, x^{\prime}{ }_{p-1} \leq x^{\prime} \leq x_{p}^{\prime} \\
\frac{\sin k_{0}\left(x^{\prime}{ }_{p+1}-x^{\prime}\right)}{\sin k_{0} \Delta x}, x_{p}^{\prime} \leq x^{\prime} \leq x^{\prime}{ }_{p+1} \\
F_{q}\left(x^{\prime}\right)=\left\{\begin{array}{l}
\frac{\sin k_{0}\left(x^{\prime}-x^{\prime}{ }_{q-1}\right)}{\sin k_{0} \Delta x}, x^{\prime}{ }_{q-1} \leq x^{\prime} \leq x^{\prime}{ }_{q} \\
\frac{\sin k_{0}\left(x^{\prime}{ }_{q+1}-x^{\prime}\right)}{\sin k_{0} \Delta x}, x^{\prime}{ }_{q} \leq x^{\prime} \leq x^{\prime}{ }_{q+1}
\end{array}\right. \\
H_{1}\left(y^{\prime}\right)=\frac{1}{\pi \sqrt{\left(W_{s} / 2\right)^{2}-\left(y^{\prime}-y_{\mathrm{s}}\right)^{2}}} \\
H_{2}\left(y^{\prime}\right)=\frac{1}{\pi \sqrt{(W / 2)^{2}-\left(y^{\prime}-y_{\mathrm{c}}\right)^{2}}}
\end{array}\right.
\end{gathered}
$$

where $a_{1 k}, a_{2 l}, e_{1 p}$, and $e_{2 q}$ are the complex expansion coefficients.

Once the current distributions on the internal posts and the slot aperture electric distribution on the crack are given, the crack characteristic signals (deviation of the current on the ammeter) of a crack in a metal can be evaluated.

\section{THEORETICAL RESULTS}

\section{Step 1: Preparation Stage}

The first step was to find the maximum current (SR) on the ammeter by adjusting the control element, $j X_{1}$, when the cutoffcavity probe was on metal with no crack. The cutoff-cavity probe of the metal was analyzed. The analysis results showed that the cutoff-cavity probe could be resonated forcefully by the control element in both SR and PR. This study focused on using SR and the ammeter as a detector.

The dimensions of the cutoff-cavity probe used in the numerical calculation were $a=40 \mathrm{~mm}, b=20 \mathrm{~mm}, c=80 \mathrm{~mm}$, $s_{1}=30 \mathrm{~mm}, s_{2}=60 \mathrm{~mm}$, and $r_{1}=r_{2}=1 \mathrm{~mm}$. The cutoff frequency was $3.75 \mathrm{GHz}$ for the dimension $a=40 \mathrm{~mm}$. The operating frequency was set to $1 \mathrm{GHz}$, which satisfied the cutoff state. The impedance of the ammeter with a diode was $Z_{M}=5.23 \mathrm{k} \Omega[5 \mathrm{k} \Omega$ (ammeter) $+230 \Omega$ (diode) $]$. The crack signals were detected by high-frequency diodes (1SS99; NEC Electronics America Inc., Santa Clara, CA, USA) connected to parasitic post \#2.
Fig. 3 presents the operating frequency of the cutoff-cavity probe as it correlates with the length of the control element. Forcedresonance occurred when the control element length is adjusted to certain values, generating SR or PR [17]. Fig. 3(a) and (b) show the control element length and reactance versus frequency, respectively. At $1 \mathrm{GHz}, \mathrm{SR}$ and $\mathrm{PR}$ occurred at $k h_{1}=1.936\left(X_{1}=\right.$ $-130.77 \Omega)$ and $k h_{1}=0.971\left(X_{1}=73.18 \Omega\right)$, respectively. At $2 \mathrm{GHz}, \mathrm{SR}$ and PR occurred at $k h_{1}=1.411\left(X_{1}=\right.$ $310.71 \Omega)$ and $k h_{1}=2.134\left(X_{1}=-79.17 \Omega\right)$, respectively. As shown in Fig. 3, for crack detection using the FRMM principle, the operating frequencies are available below the cutoff frequency of $3.75 \mathrm{GHz}$.

Fig. 4 shows the detector current (referred to as the resonance current or ammeter reading current) and the input impedance of the cutoff-cavity probe versus the length of the control element at $1 \mathrm{GHz}$ [17]. The cutoff-cavity probe with a shorting plate forcefully resonated at SR when $k h_{1}=1.936\left(X_{1}=-130.77 \Omega\right)$ for $1 \mathrm{GHz}$. As shown in Fig. 4, a variation of the input impedance of the cutoff-cavity probe at $k h_{1}=1.936$ showed a change in behavior from capacitive to inductive, which represents the SR state. Moreover, a variation of the input impedance of the cutoffcavity probe at $k h_{1}=0.971$ for $1 \mathrm{GHz}$ showed a change

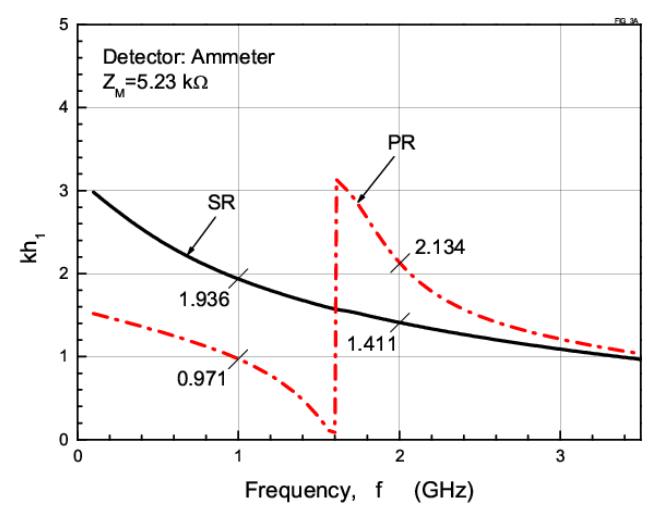

(a)

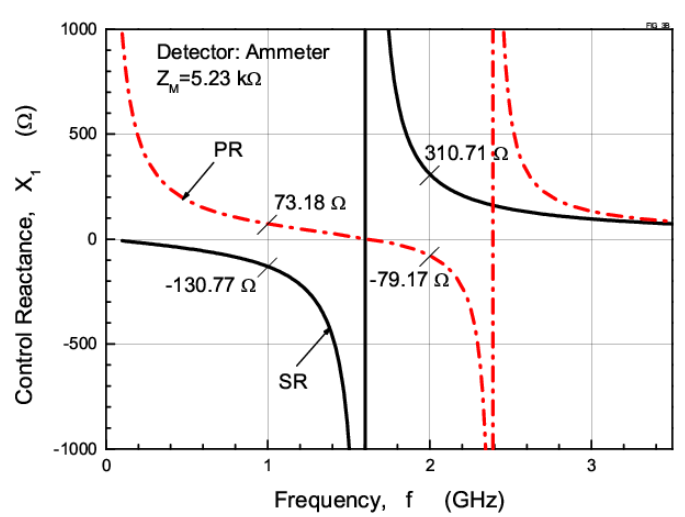

(b)

Fig. 3. Frequency characteristics of a cutoff-cavity probe: (a) control element length and (b) control reactance versus operating frequency. 


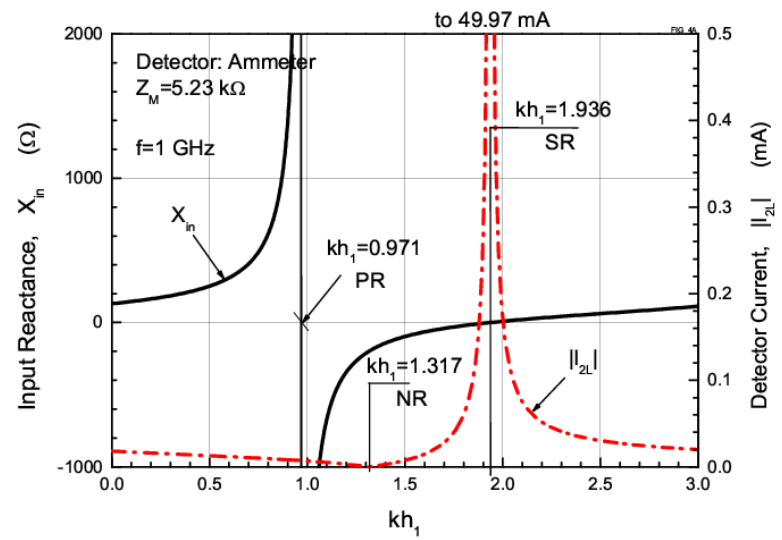

Fig. 4. Detector current and input reactance of the cutoff-cavity probe versus the control element length at $1 \mathrm{GHz}$.

from inductive to capacitive, which represents the PR state. Although it appears that the current at $k h_{1}=1.317\left(X_{1}=\right.$ $192.76 \Omega$ ) for $1 \mathrm{GHz}$ is minimized $(\approx 0 \mathrm{~mA}$ and the nonresonance $[\mathrm{NR}]$ state), this study focused only on $\mathrm{SR}$, or the maximum current.

To summarize Step 1, the preparation stage, $1 \mathrm{GHz}$ was selected as the operating frequency, and the SR stage was adopted as the reference current (maximum current). Therefore, the length of the control element was adjusted to obtain the maximum current and the control element, $k h_{1}=1.936\left(X_{1}=-130.77 \Omega\right)$, was fixed. The resonance currents were recorded as $I_{2 \mathrm{~L}}=$ $49.97 \mathrm{~mA}$ (ammeter reading), which represents the reference current or forced-resonance signal.

Fig. 5 presents the reference signals at the standoff distance from the non-crack material. There was a very rapid change in the reference signal within $0.5 \mathrm{~mm}$. A constant reference signal appeared above a $3 \mathrm{~mm}$ standoff distance. In this paper, $1 \mathrm{~mm}$ was chosen as the reference value of the standoff distance.

\section{Step 2: Crack Detection Stage}

When the cutoff-cavity probe moves over a crack on a metal

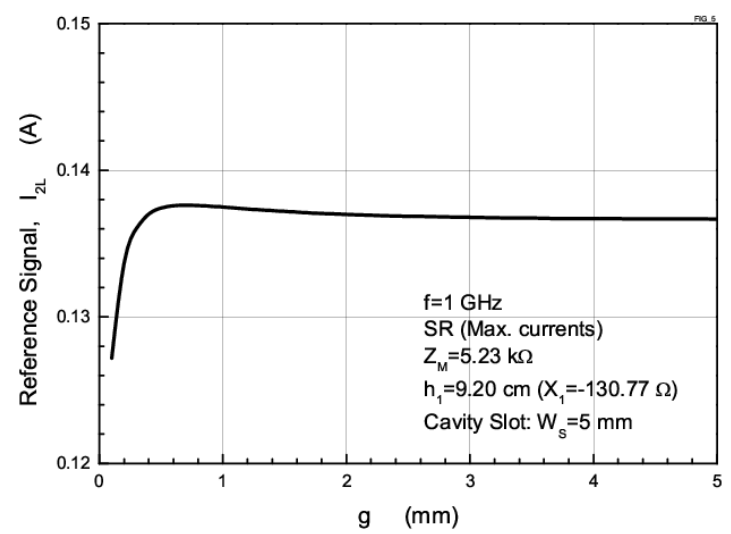

Fig. 5. Reference signals at standoff distance from the non-crack material. sturface (lift-off of $1 \mathrm{~mm}$ ), as shown in Fig. 2, its reference current or forced-resonance signal (maximum current) changes. The reactance value of the control element as a reactance element, $X_{1}=$ $-130.77 \Omega\left(k h_{1}=1.936\right)$, for $1 \mathrm{GHz}$, obtained in Step 1 , was entered into (3) to determine the change in current detected on the ammeter when there is a crack. The current expansion modes $K=L=29$ and crack aperture electric field expansion mode $Q=$ 19 were used to calculate the convergence results.

As shown in Fig. 2, the scanning position $T$ indicates the position of the cutoff-cavity probe relative to a crack position. The crack signal refers to the variations in the detector current, as a function of scanning position $T$, which is obtained when the cutoff -cavity probe detects a surface crack in the metal. The difference in the ammeter currents (resonance currents) in Steps 1 and 2 is referred to as the characteristic crack signal.

Fig. 6 shows the crack characteristic signals (normalized crack signals, with respect to the value of the forced-resonance signal in Step 1) recorded at $1 \mathrm{GHz}$ for various crack widths. The crack length $L$ is in the $x$-direction, classifying it as an $x$-directed crack. The cutoff-cavity probe was scanned linearly along the $y$-axis in search of an $x$-directed crack. As shown in Fig. 6, the detector value did not change significantly when the $x$-directed crack was

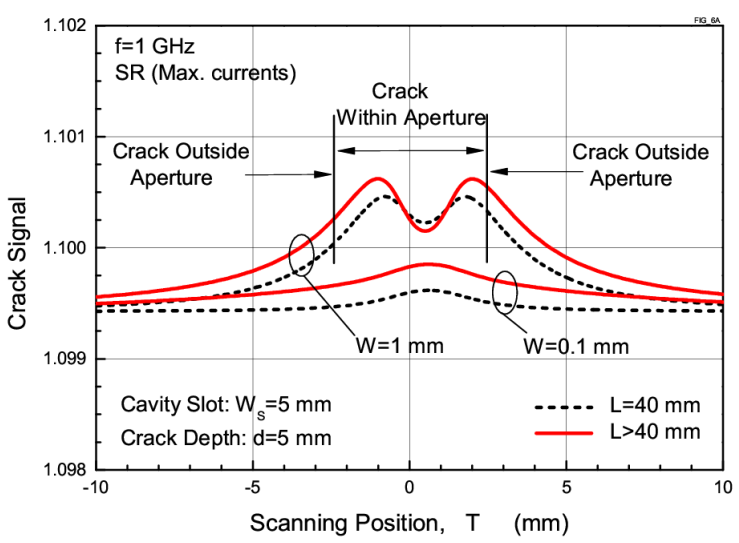

(a)

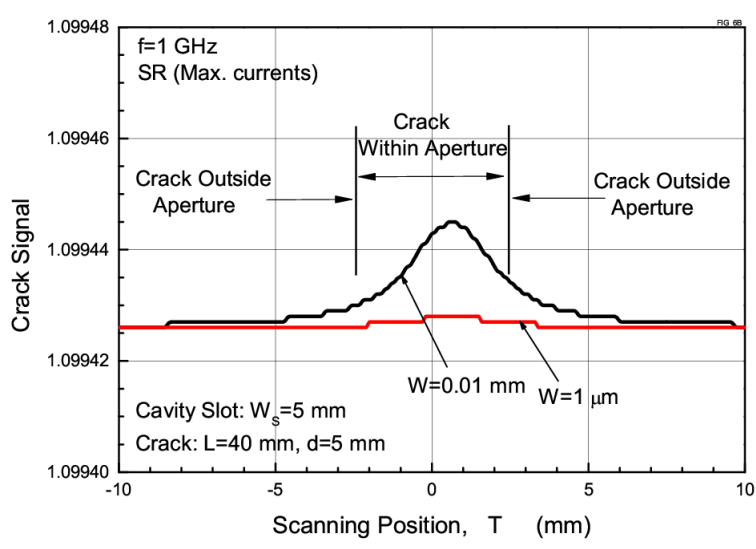

(b)

Fig. 6. Normalized crack signals for various crack widths: (a) $W$ $=1 \mathrm{~mm}$ and $0.1 \mathrm{~mm}$ and (b) $W=0.01 \mathrm{~mm}$ and $1 \mu \mathrm{m}$. 
outside the narrow slot of the cutoff cavity; the absence of a crack results in a fairly constant signal. As expected, for a $y$-directed crack (where the crack length is in the $y$-direction), the detector value did not change while the crack was inside the narrow slot of the cutoff cavity. A crack inside the narrow slot did not prompt a change in the resonance current because there were nopropagation modes.

Fig. 6(a) shows the crack characteristic signals for crack widths when the crack is located in the $x$-direction using non-contact (standoff or remote) detection. The detector output signals were dependent on the crack widths. In addition, Fig. 6(a) also shows that when the crack length extends beyond the cavity-slot, $L>$ $L_{S}(=40 \mathrm{~mm})$, there will be a considerable perturbation in the crack signal. Fig. 6(b) shows the crack characteristic signals for very thin crack widths when the crack is located in the $x$-direction using non-contact (standoff or remote) detection. The crack signals were dependent on very thin crack widths. The crack widths were small compared to the wavelength, $3.3 \times 10^{-5} \lambda$ (for $1 \mu \mathrm{m}$ ) to $3.3 \times 10^{-2} \lambda$ (for $1 \mathrm{~mm}$ ), when the operating frequency was $1 \mathrm{GHz}$. The results showed that the crack signals for wide and narrow cracks were detected at a single operating frequency.

Fig. 7 shows the crack characteristic signals for various wide crack widths, $W>W_{S}(=5 \mathrm{~mm})$, when the crack was located in the $x$-direction using non-contact detection. The crack signals were also detected and changed for the wide crack width.

Fig. 8 shows the crack characteristic signals for various crack depths when the crack was located in the $x$-direction using noncontact detection. The cutoff-cavity probe was scanned linearly along the $y$-axis in search of an $x$-directed crack. The detector output signals were dependent on the various crack depths. When the operating frequency was $1 \mathrm{GHz}$, the crack depths were smaller than the wave length, $0.033 \lambda$ (for $1 \mathrm{~mm}$ ) to $0.167 \lambda$ (for $5 \mathrm{~mm}$ ). The results showed that crack signals for deep and shallow cracks were detected at a single operating frequency. Overall, the operating frequency used in conventional microwave methods for

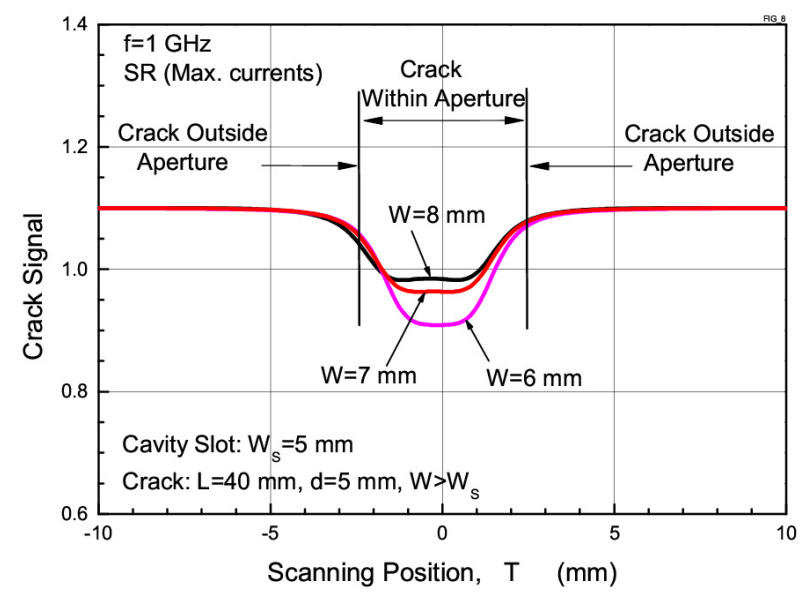

Fig. 7. Normalized crack signals for various wide crack widths.

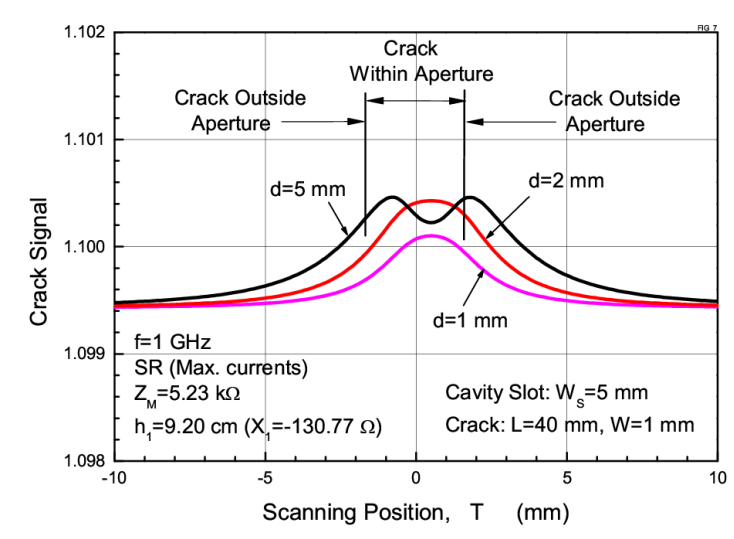

Fig. 8. Normalized crack signals for various crack depths.

crack detection depends on the dimensions of the crack, but the FRMM can be used to detect cracks with different sizes using a single frequency.

A rapid change in the amplitude of the resonance current occurred when the crack moved within the narrow slot of the cutoff cavity. The amplitude of the resonance current remained fairly constant when the crack was outside the cutoff-cavity probe, as shown in Figs. 6 and 7.

Fig. 9 shows the crack signals at the standoff distance (air gap) from the crack center. There was a very rapid change in the crack signal within $0.5 \mathrm{~mm}$. A constant crack signal appeared above a $0.7 \mathrm{~mm}$ standoff distance. Therefore, remote crack detection can be used within $0.7 \mathrm{~mm}$.

\section{EXPERIMENTS}

Fig. 10 presents a photograph of the experimental setup. The cutoff cavity-backed narrow slot probe was made with copper and plated with gold. The control reactance element was constructed using a semi-rigid coaxial cable with a short end. The dimensions of the cutoff-cavity probe used in the experiments were $a=40$ $\mathrm{mm}, b=20 \mathrm{~mm}, c=80 \mathrm{~mm}, s_{1}=30 \mathrm{~mm}, s_{2}=60 \mathrm{~mm}$, and $r_{1}=r_{2}=1 \mathrm{~mm}$. A $1 \mathrm{SS} 99$ (NEC) diode with an ammeter $(20$

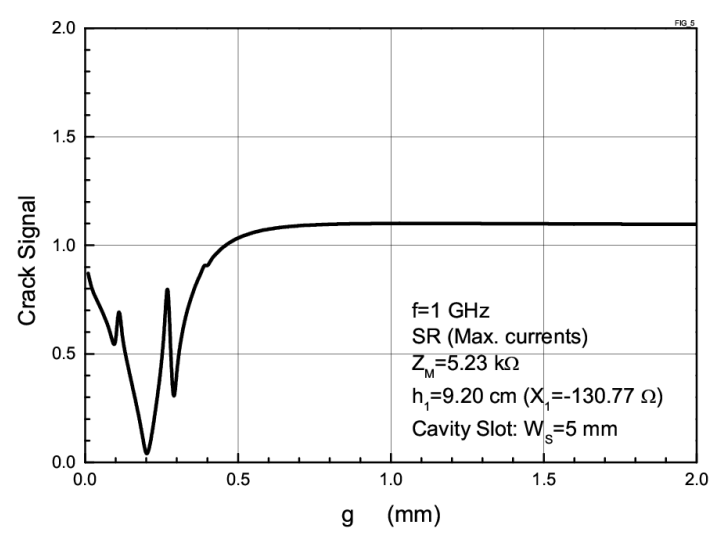

Fig. 9. Normalized crack signals vs. air gap from the crack center. 


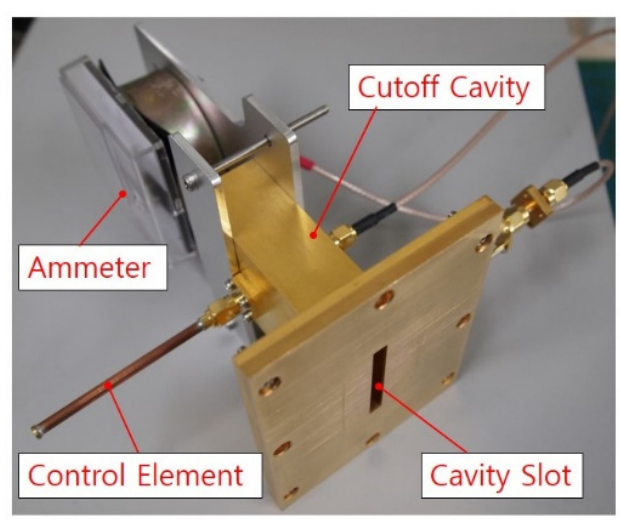

(a)

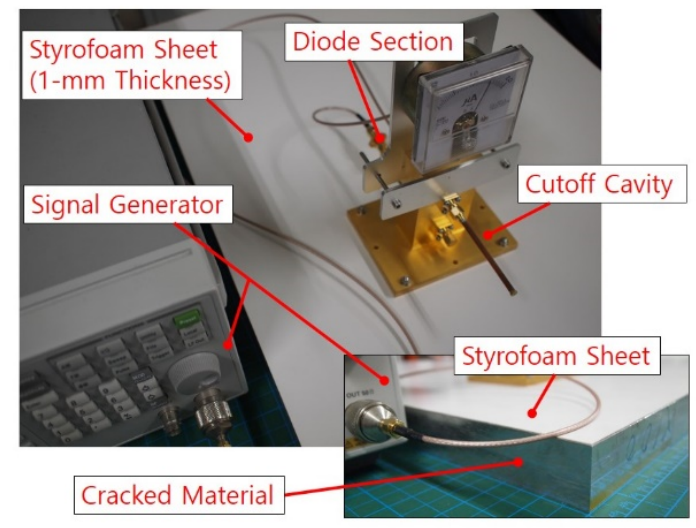

(b)

Fig. 10. (a) Cutoff cavity-backed narrow slot probe and (b) the experimental setup.

$\mu \mathrm{A}$ range) was connected to the parasitic post. The operating frequency was $1 \mathrm{GHz}$, and the control element was made of a semi-rigid coaxial cable. The length of the control element was adjusted to obtain the forced resonance in the cutoff-cavity probe with a shorting plate. The cracked material used in the experiment was a thick aluminum plate with a thickness of $50 \mathrm{~mm}$. The dimensions of the crack made on the thick aluminum plate were $L=40 \mathrm{~mm}, W=1 \mathrm{~mm}$, and $d=2 \mathrm{~mm}$.

As the first step, the SR was produced by the control reactance element on the non-crack material and the signal output was recorded by the ammeter. As the second step, the cutoff-cavity probe scanned for cracks in the thick aluminum. A gradually change in the amplitude of the cavity resonance current occured when a crack appeared inside the cutoff-cavity slot aperture, as shown in Fig. 11.

Fig. 11 shows the calculated and measured crack characteristic signals for the $x$-directed crack. The measured crack signal was detected at a standoff distance of $1 \mathrm{~mm}$ using the cutoff-cavity probe. A 1-mm Styrofoam sheet was used to maintain a constant lift-off distance at the constant standoff distance from the cracked material. The characteristic crack signals were detected using a

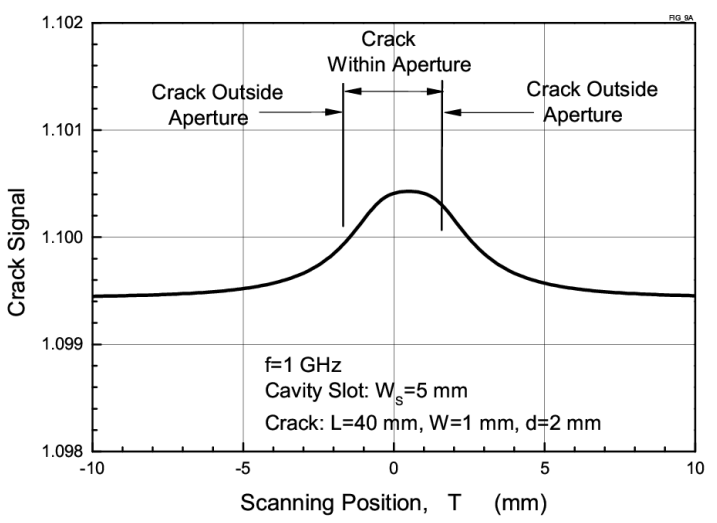

(a)

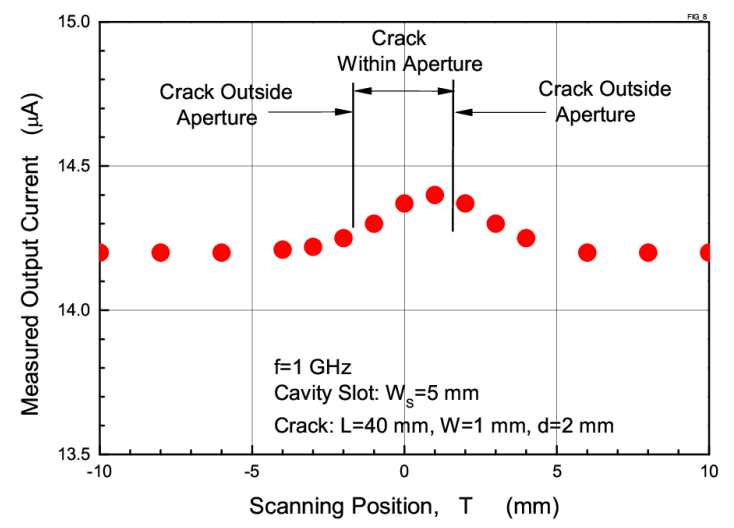

(b)

Fig. 11. Measured crack signals: (a) calculated and (b) measured.

single microwave frequency and SR (forced-resonance) in the cutoff cavity-backed narrow slot.

\section{CONCLUSION}

Non-contact (remote or lift-off) crack detection using a FRMM through a cutoff cavity-backed narrow slot probe was demonstrated. The crack characteristic signals of the FRMM were evaluated theoretically and compared with the measured results. A thin-wire approximation with Galerkin's method of moments was used to express the crack aperture field. Because the forced resonance of a cutoff cavity can be achieved by adjusting an external control element, this technique has the potential to enable the sensitive detection of cracks in metal at microwave frequencies. Future studies will investigate surface crack detection in filled and dielectric material coatings.

This research was supported by the Basic Science Research Program through the National Research Foundation of Korea (NRF), which is funded by the Ministry of Education (No. NRF-2019R1D1A1A09058357). 


\section{REFERENCES}

[1] K. G. Boving, NDE Handbook: Non-Destructive Examination Methods for Condition Monitoring. London, UK: Butterworths, 1989.

[2] R. Zoughi, Microwave Non-Destructive Testing and Evaluation Principles. Dordrecht, The Netherlands: Kluwer Academic Publishers, 2000.

[3] A. J. Bahr, Microwave Nondestructive Testing Methods. New York, NY: Gordon and Breach, 1982.

[4] C. Y. Yeh and R. Zoughi, "A novel microwave method for detection of long surface cracks in metals," IEEE Transactions on Instrumentation and Measurement, vol. 43, no. 5, pp. 719$725,1994$.

[5] C. Yeh and R. Zoughi, "Microwave detection of finite surface cracks in metals using rectangular waveguides," Research in Nondestructive Evaluation, vo. 6, pp. 35-55, 1994.

[6] R. Zoughi, S. I. Ganchev, and C. Huber, "Microwave measurement-parameter optimization for detection of surface breaking hairline cracks in metals," Nondestructive Testing and Evaluation, vol. 14, no. 5, pp. 323-337, 1998.

[7] H. H. Park, Y. H. Cho, and H. J. Eom, "Surface crack detection using flanged parallel-plate waveguide," Electronics Letters, vol. 37, no. 25, pp. 1526-1527, 2001.

[8] R. Zoughi and S. Kharkovsky, "Microwave and millimetre wave sensors for crack detection," Fatigue \& Fracture of Engineering Materials \& Structures, vol. 31, no. 8, pp. 695-713, 2008.

[9] K. M. Donnell, A. McClanahan, and R. Zoughi, "On the crack characteristic signal from an open-ended coaxial probe," IEEE Transactions on Instrumentation and Measurement, vol. 63, no. 7, pp. 1877-1879, 2014.

[10] J. Kerouedan, P. Queffelec, P. Talbot, C. Quendo, S. De Blasi, and A. Le Brun, "Detection of micro-cracks on metal surfaces using near-field microwave dual-behavior resonator filters," Measurement Science and Technology, vol.19, no. 10, article no. 105701, 2008. https://doi.org/10.1088/0957-0233/19/10/10 5701

[11] A. Husain and E. A. Ash, "Microwave scanning microscopy for non-destructive testing," in Proceedings of 1975 5th European Microwave Conference, Hamburg, Germany, 1975, pp. 213-217.

[12] A. M. Albishi and O. M. Ramahi, "Microwaves-based high sensitivity sensors for crack detection in metallic materials," IEEE Transactions on Microwave Theory and Techniques, vol. 65, no. 5, pp. 1864-1872, 2017.

[13] A. M. Albishi, M. S. Boybay, and O. M. Ramahi, "Complementary split-ring resonator for crack detection in metallic surfaces," IEEE Microwave and Wireless Components Letters, vol. 22, no. 6, pp. 330-332, 2012.

[14] T. Yun and S. Lim, "High-Q and miniaturized complementary split ring resonator-loaded substrate integrated waveguide microwave sensor for crack detection in metallic materials," Sensors and Actuators A: Physical, vol. 214, pp. 25-30, 2014.

[15] M. U. Memon and S. Lim, "Review of electromagneticbased crack sensors for metallic materials (recent research and future perspectives) ," Metals, vol. 6, no. 8, article no. 172, 2016. https://doi.org/10.3390/met6080172

[16] K. C. Kim, J. Y. Kwon, and N. W. Kang, "A novel forcedresonance microwave method to detect surface cracks in metal," IEICE Electronics Express, vol. 13, no. 17, article no. 20160715,2016. https://doi.org/10.1587/elex.13.20160715

[17] K. C. Kim, J. W. Kim, J. Y. Kwon, and N. W. Kang, "Characteristics of a cutoff cavity probe applicable to crack detection using the forced resonance microwave method," Journal of Electromagnetic Engineering and Science, vol. 20, no. 4, pp. 285-292, 2020. 


\section{Ki-Chai Kim}

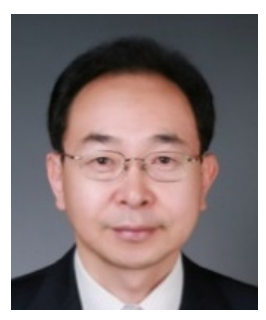

received a B.S. degree in electronic engineering from Yeungnam University, Korea, in 1984, and M.S. and Doctor of Engineering degrees in electrical engineering from Keio University, Japan, in 1986 and 1989, respectively. He was a senior researcher at Korea Standards Research Institute, Daedok Science Town, Korea, until 1993, working in electromagnetic compatibility. From 1993 to 1995, he was an Associate Professor at Fukuoka Institute of Technology, Fukuoka, Japan. Since 1995, he has been with Yeungnam University, Gyeongsan, Korea, where he is currently a professor in the Department of Electrical Engineering. He received the 1988 Young Engineer Award from the Institute of Electronics, Information and Communication Engineers (IEICE) of Japan and received a Paper Presentation Award in 1994 from the Institute of Electrical Engineers (IEE) of Japan. Prof. Kim served as President of the Korea Institute of Electromagnetic Engineering and Science (KIEES) in 2012. His research interests include EMC/EMI antenna evaluation, electromagnetic penetration problems in slots, and applications of electromagnetic fields and waves.

\section{Jong-Woo Kim}

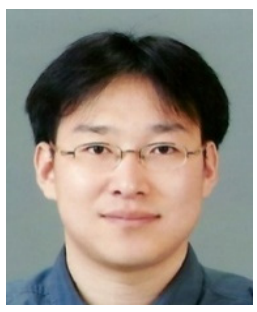

was born in Seoul, Korea. He received a M.S. degree in electronic engineering from Yeungnam University, Korea2012. He was an assistant manager in the reliability department of Daesung Corporation, Ansan, Korea, from 2004 to 2006, working in electromagnetic compatibility. He was a team manager at KOMERI (Korea Marine Equipment Research Institute) from 2007 to 2015, working on an electromagnetic compatibility test team. He is currently a department manager at KOMERI, working in the marine electronics testing and certification department.
Jae-Yong Kwon

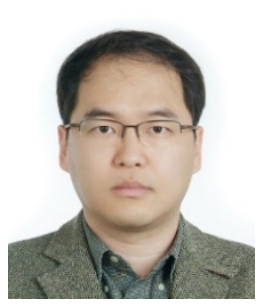

received a B.S. degree in electronics from Kyungpook National University, Daegu, in 1995, and M.S. and $\mathrm{Ph} . \mathrm{D}$. degrees in electrical engineering from the Korea Advanced Institute of Science and Technology, Daejeon, Korea, in 1998 and 2002, respectively. He was a visiting scientist at the National Institute of Standards and Technology (NIST) in Boulder, CO, USA, in 2010. From 2002 to 2005, he was a senior research engineer at the Devices and Materials Laboratory at the LG Electronics Institute of Technology, Seoul, Korea. He has been with the Korea Research Institute of Standards and Science, Daejeon, Korea, since 2005, where he is currently the head of the Electromagnetic Wave Metrology Group and a principal research scientist. Since 2013, he has been a professor of measurement science with the University of Science and Technology, Daejeon, Korea. His current research interests include electromagnetic power, impedance, and antenna measurement. Dr. Kwon is a Korea Institute of Electromagnetic Engineering and Science (KIEES) life member and IEICE member.

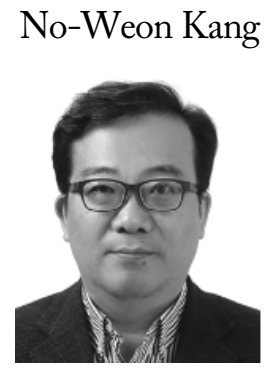

received his B.S., M.S., and Ph.D. degrees in electrical engineering from Seoul National University, Seoul, South Korea, in 1991, 1994, and 2004, respectively. His Ph.D. degree was focused on a numerical analysis of the finite-difference time-domain method, in particular, an algorithm that reconstructs the complex permittivity profile of unknown scatterers. He was with the Electromagnetic Apparatus Laboratory, LG Industrial Systems, Seoul, from 1994 to 1999, where he was involved in the static magnetic field analysis of electrical apparatus. He has been with the Korea Research Institute of Standards and Science, Daejeon, South Korea, since 2004, where he is currently the director of the Division of Physical Metrology and a principal research scientist in electromagnetic metrology. His current research interests include electromagnetic field strength, antenna measurement standards, electromagnetic interference/electromagnetic interference issues, and electro-optic sensors. 